\title{
Expression profiling of marker genes responsive to the defence-associated phytohormones salicylic acid, jasmonic acid and ethylene in Brachypodium distachyon
}

Yusuke Kouzai ${ }^{1}$, Mamiko Kimura', Yurie Yamanaka ${ }^{1}$, Megumi Watanabe ${ }^{1}$, Hidenori Matsui ${ }^{1}$, Mikihiro Yamamoto', Yuki Ichinose', Kazuhiro Toyoda', Yoshihiko Onda², Keiichi Mochida ${ }^{2}$ and Yoshiteru Noutoshi ${ }^{1 *}$

\begin{abstract}
Background: Brachypodium distachyon is a promising model plants for grasses. Infections of Brachypodium by various pathogens that severely impair crop production have been reported, and the species accordingly provides an alternative platform for investigating molecular mechanisms of pathogen virulence and plant disease resistance. To date, we have a broad picture of plant immunity only in Arabidopsis and rice; therefore, Brachypodium may constitute a counterpart that displays the commonality and uniqueness of defence systems among plant species. Phytohormones play key roles in plant biotic stress responses, and hormone-responsive genes are used to qualitatively and quantitatively evaluate disease resistance responses during pathogen infection. For these purposes, defence-related phytohormone marker genes expressed at time points suitable for defence-response monitoring are needed. Information about their expression profiles over time as well as their response specificity is also helpful. However, useful marker genes are still rare in Brachypodium.
\end{abstract}

Results: We selected 34 candidates for Brachypodium marker genes on the basis of protein-sequence similarity to known marker genes used in Arabidopsis and rice. Brachypodium plants were treated with the defence-related phytohormones salicylic acid, jasmonic acid and ethylene, and their transcription levels were measured 24 and $48 \mathrm{~h}$ after treatment. Two genes for salicylic acid, 7 for jasmonic acid and 2 for ethylene were significantly induced at either or both time points. We then focused on 11 genes encoding pathogenesis-related (PR) 1 protein and compared their expression patterns with those of Arabidopsis and rice. Phylogenetic analysis suggested that Brachypodium contains several PR1-family genes similar to rice genes. Our expression profiling revealed that regulation patterns of some $P R 1$ genes as well as of markers identified for defence-related phytohormones are closely related to those in rice.

Conclusion: We propose that the Brachypodium immune hormone marker genes identified in this study will be useful to plant pathologists who use Brachypodium as a model pathosystem, because the timing of their transcriptional activation matches that of the disease resistance response. Our results using Brachypodium also suggest that monocots share a characteristic immune system, defined as the common defence system, that is different from that of dicots.

Keywords: Brachypodium distachyon, Phytohormone, Salicylic acid, Jasmonic acid, Ethylene, Plant disease resistance, Defense mechanism, Immunity system, Marker gene

\footnotetext{
* Correspondence: noutoshi@okayama-u.ac.jp

${ }^{1}$ Graduate School of Environmental and Life Science, Okayama University,

Kita-ku, Okayama, Japan

Full list of author information is available at the end of the article
}

\section{Biomed Central}

(c) 2016 Kouzai et al. Open Access This article is distributed under the terms of the Creative Commons Attribution 4.0 International License (http://creativecommons.org/licenses/by/4.0/), which permits unrestricted use, distribution, and reproduction in any medium, provided you give appropriate credit to the original author(s) and the source, provide a link to the Creative Commons license, and indicate if changes were made. The Creative Commons Public Domain Dedication waiver (http://creativecommons.org/publicdomain/zero/1.0/) applies to the data made available in this article, unless otherwise stated. 


\section{Background}

To counteract various pathogens in the field, plants mainly protect themselves with a two-layered immune system. Using cell surface-localised receptors, plants recognise pathogen- or microbe-associated molecular patterns (PAMPs or MAMPs), which are structurally conserved molecules in a broad range of microorganisms, that may include products of housekeeping genes or cell wall components and induce the expression of defence-related genes. This system provides basal resistance called PAMP/MAMP-triggered immunity (PTI/ MTI) [1]. For the successful infection of host plants, pathogens use a few dozen effector proteins as a weapon to suppress PTI. Plants can directly or indirectly sense these effectors by cytoplasmic nucleotide-binding domain- and leucine-rich repeat-containing (NLR) immune sensors and activate a strong resistance response called effector-triggered immunity (ETI) that is effective against pathogens [2]. ETI is often accompanied by hypersensitive responses including programmed cell death of infected regions containing pathogens. In a battery of these immune responses, the phytohormone salicylic acid (SA) plays important roles in mediating signal transduction. Another phytohormone, ethylene (ET), is also required to maintain the level of patternrecognition receptors in PTI [3]. This defence system effectively functions to block biotrophic or hemibiotrophic pathogens. Plants have another defence system relying on the phytohormones jasmonic acid (JA) and ET to combat necrotrophic pathogens and insects [4].

To characterise plant responses to a given pathogen, the production of phytohormones may be appropriate indicators in addition to the phenotypic observation of lesion formation. However, in rice and barley, endogenous SA levels are not increased, even in response to incompatible pathogens, unlike the case of well-studied dicotyledonous model plants such as Arabidopsis thaliana and tobacco [5-7]. Alternatively, phytohormone production can be substituted with the expression profiling of phytohormone-responsive marker genes. This approach provides information about the time, strength and kind of responses provoked in plants. For example, PATHOGENESIS-RELATED1 (PR1) and PDF1.2 (PLANT DEFENSIN1.2) are used as markers for SA and JA or ET, respectively, in Arabidopsis [8, 9]. In model plants, genes considered to be involved in phytohormone biosynthesis or signalling are also used as markers $[9,10]$.

Brachypodium distachyon (purple false brome) is a grass plant of the Pooideae subfamily, which includes economically important crops such as wheat, barley, rye and oats. Owing to its small stature, short lifecycle, selffertility and small diploid genome, Brachypodium can be an experimental model plant for studies of grasses including cereals and biomass crops [11]. A whole- genome sequence of $B$. distachyon cultivar $\mathrm{Bd} 21$ was obtained [12] and a database of full-length cDNA (FLcDNA) is available [13]. Recently, the superiority of this plant as a model for Triticeae crops has been shown by the similarities of morphological property and by the commonalities of metabolic profile [14]. For investigation of immunity as one of the important traits in agriculture, infectivity on Brachypodium of various pathogens threatening world crop cultivation has been verified so far [15]. For example, Fusarium graminearum and Magnaporthe oryzae, causal fungi of wheat Fusarium head blight and rice blast, respectively, are pathogenic to Brachypodium [16, 17]. Bacterial pathogen Xanthomonas oryzae pv. oryzae and a pathogenic virus Panicum mosaic virus are also virulent to Brachypodium $[18,19]$. Thus, Brachypodium may be a useful platform for investigating both crop pathogen virulence and plant immune response at the molecular level.

Several phytohormone marker genes have been used to date to characterise resistance responses in Brachypodium, but the number of markers is still limited and inadequate. Most recently, a comprehensive transcriptome analysis of various phytohormones in Brachypodium using RNA-seq technology was performed and phytohormone-responsive genes were identified [20]. In that study, hormone treatment was for $1 \mathrm{~h}$ for JA and ET and $3 \mathrm{~h}$ for SA using young seedlings. For investigations of plant-microbe interaction, for each immune phytohormone, several sets of marker genes upregulated at appropriate time points during infection process are needed.

For the present study, we chose candidates for Brachypodium genes responsive to SA, JA and ET based on the similarity of protein sequences to known marker genes used in Arabidopsis and rice and analysed their transcriptional activation by each hormone at 24 and $48 \mathrm{~h}$ after treatment. As a result, we identified at least 2 marker genes for each hormone. In addition, we compared the constitutions and expression profiles of PR1 family genes from Arabidopsis, rice and Brachypodium, finding that $B$. distachyon possesses immunity mechanisms similar to those of rice but not of Arabidopsis.

\section{Results and discussions}

Identification of candidates for marker genes responsive to defence-related phytohormones in Brachypodium

We selected candidates for phytohormone-responsive genes in Brachypodium, based on the similarities to experimentally validated markers in rice, barley and Arabidopsis. For BdTARL1 and BdTARL2 genes in $B$. distachyon, their responsiveness to 1-aminocyclopropane -1-carboxylic acid (ACC), a precursor of ET, has already been demonstrated [21]. The protein sequences of these selected genes were used as queries in a BLAST search 
against the RIKEN Brachypodium FLcDNA database, and the resulting hits with high similarity were identified as potential markers [13, 22]. Twenty-three genes were tested for transcriptional inductions during treatment with SA, JA or ET (Table 1).

Whole Brachypodium seedlings were treated with water as a mock treatment, $1 \mathrm{mM}$ sodium salicylate, $100 \mu \mathrm{M}$ methyl jasmonate (MeJA) or $100 \mu \mathrm{M}$ ethephon for 24 or $48 \mathrm{~h}$. Total RNAs were extracted from the frozen leaf samples and subjected to cDNA synthesis. The mRNA levels of the candidate genes were analysed by quantitative reverse-transcription polymerase chain reaction (qRT-PCR) using specific primers designed with the Primer3 program [23]. The responsiveness of each gene is summarised in Table 2. Among these genes, 8 were significantly induced by a phytohormone, whereas the remaining 15 genes showed no change in expression.

To obtain SA markers in Brachypodium, we focused on genes encoding WRKY-domain containing transcription factors. In rice, OsWRKY45, 62 and 76 genes were induced by SA treatment, and all of them were shown to participate in the immune response [24-26]. Among them, OsWRKY45 plays a central role in SA signalling, together with OsNPR1, and mediates SA-induced disease resistance [24]. Using RNA-seq technology in rice, transcriptional upregulation of OsWRKY45 was detected at $24 \mathrm{~h}$ after inoculation of both compatible and incompatible strains of $M$. oryzae [27]. Its induction by SA was also observed $12 \mathrm{~h}$ after SA treatment [24]. In Brachypodium, two genes, Bradi2g30695 and Bradi2g44270, were

Table 1 Candidate marker genes selected in this study for SA, JA and ET in Brachypodium

\begin{tabular}{|c|c|c|c|c|c|}
\hline $\mathrm{ID}$ & Name & Description in database & Rice homolog & Arabidopsis homolog & Ref \\
\hline \multicolumn{6}{|l|}{ SA-related genes } \\
\hline Bradi2g05870 & NPR1 & Regulatory protein NPR1-like & OsNPR1:Os01g0194300 & NPR1 : At1g64280 & [24] \\
\hline Bradi2g30695 & WRKY45-1 & Uncharacterized protein & OsWRKY45-1 : Os05g0322900 & AtWRKY70 : At3g56400 & [24] \\
\hline Bradi2g44270 & WRKY45-2 & WRKY transcription factor 70-like & OsWRKY45-1 : Os05g0322900 & AtWRKY70 : At3g56400 & [24] \\
\hline Bradi4g35356 & SAGT1 & UDP-glycosyltrasferase 74 F1-like & OsSGT1 : Os09g0518200 & UGT superfamily : At1g05675 & [29] \\
\hline Bradi2g22410 & AGA & $\begin{array}{l}\text { Alanine-glyoxylate aminotransferase } 2 \\
\text { homolog } 3\end{array}$ & Osh36: Os05g0475400 & AtPYD4 : At3g08860 & [29] \\
\hline Bradilg53527 & UGT76-1 & UDP-glycosyltrasferase 76C2-like & no symbol: Os07g0241500 & UGT76B1 : At3g11340 & [30] \\
\hline Bradilg53540 & UGT76-2 & UDP-glycosyltrasferase 76C2-like & no symbol : Os07g0241500 & UGT76B1 : At3g11340 & [30] \\
\hline Bradi1g53550 & UGT76-3 & UDP-glycosyltrasferase 76 F1-like & no symbol : Os07g0241500 & UGT76B1 : At3g11340 & [30] \\
\hline Bradi4g41410 & UGT76-4 & UDP-glycosyltrasferase 76C2-like & no symbol : Os07g0241500 & UGT76B1 : At3g11340 & [30] \\
\hline Bradilg11940 & UGT74-1 & Indole-3-acetate beta-glucosyltransferase-like & OsIAGLU : Os03g0693600 & UGT74F2 : At2G43820 & [30] \\
\hline Bradi4g35350 & UGT74-2 & UDP-glycosyltrasferase 74 F2-like & no symbol : Os09g0517900 & UGT74F2 : At2G43820 & [30] \\
\hline Bradi5g03380 & UGT74-3 & UDP-glycosyltrasferase 74 F2-like & no symbol : Os04g0206500 & UGT74F2 : At2G43820 & [30] \\
\hline \multicolumn{6}{|l|}{ JA-related genes } \\
\hline Bradi1g69330 & AOS & Allene oxide synthase 2 -like & OsAOS2 : Os03g0225900 & AtAOS2 : At5g42650 & [32-34] \\
\hline Bradi1g11670 & LOX & Linoleate 95-lipoxygenase 4-like & OsLOX1 : Os03g0700700 & AtLOX5 : At3g22400 & {$[32-34]$} \\
\hline \multicolumn{6}{|l|}{ ET-related genes } \\
\hline Bradi2g52370 & ERF & Ethylene-responsive transcription factor 4-like & OsERF3 : Os01g0797600 & AtERF9 : At5g44210 & [43] \\
\hline Bradilg63780 & EIN3 & Ethylene insensitive 3-like & no symbol : Os03g0324300 & AtEIN3 : At3g20770 & [42] \\
\hline Bradi1g49966 & ACC & Aminotransferase ACS10-like & OsACS6 : Os06g0130400 & AtACS10 : At1g62960 & [44] \\
\hline Bradi2g34400 & TAR1 & $\begin{array}{l}\text { Tryptophan aminotransferase-related protein } \\
\text { 2-like }\end{array}$ & OsTAR1 : Os05g0169300 & AtTAR2 : At4g24670 & [21] \\
\hline Bradi2g04290 & TAR2 & $\begin{array}{l}\text { Tryptophan aminotransferase-related protein } \\
\text { 2-like }\end{array}$ & OsTAR1 : Os05g0169300 & AtTAR2 : At4g24670 & [21] \\
\hline Bradi3g37300 & $4 \mathrm{CL}$ & 4-Coumarate:CoA ligase 5-like & Os4CL5: Os08g0448000 & At4CL1 : At1g51680 & {$[35,37-39]$} \\
\hline Bradi3g48840 & PAL & Phenylalanine ammonia-lyase-like & OsPAL1 : Os02g0627100 & AtPAL1 : At2g37040 & {$[35,37-39]$} \\
\hline Bradilg33540 & PR5 & Thaumatin-like protein-like & no symbol: Os06g0691200 & no symbol : At1g73620 & {$[45,47]$} \\
\hline Bradi4g05040 & PBZ1 & Major allergen Api g 1-like & PBZ1-like : Os 12g0555000 & no hit & {$[45,47]$} \\
\hline
\end{tabular}

Twenty-three Brachypodium genes were identified by similarity search using known phytohormone marker genes of rice or Arabidopsis as queries. Gene IDs, relationships to phytohormone, expedient names without functional confirmation, descriptions in the database, corresponding homologs in rice or Arabidopsis, and references are listed 
Table 2 Transcriptional responses of tested genes to SA, JA and ET

\begin{tabular}{|c|c|c|c|c|}
\hline \multirow[b]{2}{*}{ ID } & \multirow[b]{2}{*}{ Name } & \multicolumn{3}{|c|}{ Inducibility in Brachypodium } \\
\hline & & SA & $\mathrm{JA}$ & ET \\
\hline Bradi2g05870 & NPR1 & - & - & - \\
\hline Bradi2g30695 & WRKY 45-1 & ++ & - & - \\
\hline Bradi2g44270 & WRKY45-2 & ++ & $+(48 \mathrm{~h})$ & $+(48 h)$ \\
\hline Bradi4g35356 & SAGT1 & - & - & - \\
\hline Bradi2g22410 & $A G A$ & - & - & - \\
\hline Bradilg53527 & UGT76-1 & - & - & - \\
\hline Bradilg53540 & UGT76-2 & - & - & - \\
\hline Bradilg53550 & UGT76-3 & - & - & - \\
\hline Bradi4g41410 & UGT76-4 & - & - & + \\
\hline Bradilg11940 & UGT74-1 & - & - & - \\
\hline Bradi4g35350 & UGT74-2 & - & - & - \\
\hline Bradi5g03380 & UGT74-3 & - & - & - \\
\hline Bradilg69330 & AOS & - & ++ & - \\
\hline Bradilg11670 & LOX & - & + & - \\
\hline Bradi2g52370 & $E R F$ & - & - & - \\
\hline Bradilg63780 & EIN3 & - & - & - \\
\hline Bradilg49966 & $A C C$ & - & - & - \\
\hline Bradi2g34400 & TAR1 & - & - & - \\
\hline Bradi2g04290 & TAR2 & - & - & + \\
\hline Bradi3g37300 & $4 C L$ & $+(48 \mathrm{~h})$ & ++ & $+(48 \mathrm{~h})$ \\
\hline Bradi3g48840 & PAL & - & ++ & - \\
\hline Bradilg33540 & PR5 & - & - & - \\
\hline Bradi4g05040 & PR10(PBZ1) & - & - & - \\
\hline
\end{tabular}

Expression of 23 Brachypodium candidate genes was evaluated in 3-4 weekold plants at 24 and $48 \mathrm{~h}$ after treatment with $\mathrm{SA}, \mathrm{JA}$ or $\mathrm{ET}$, and the results are summarised. The expression levels of each gene were determined by qRT-PCR analysis. ++, genes significantly induced more than 10-fold compared to mock treatment; +, genes significantly induced more than 2 -fold compared to mock treatment, - , not induced. Experiments were performed at least three times with similar results and a representative result is shown

found, whose deduced protein sequences showed high similarity (49 and $50 \%$ identity, respectively) to OsWRKY45 throughout their lengths (Additional file 1: Figure S1). As shown in Fig. 1, transcription of these genes was upregulated by SA at $24 \mathrm{~h}$ after treatment and their expression levels were more increased at $48 \mathrm{~h}$. Kakei et al. also reported that Bradi2g44270 and Bradi2g30695 were induced at $3 \mathrm{~h}$ after treatment with $100 \mu$ M SA [20]. For Bradi2g44270, 9.9- and 4.8-fold expression changes were also detected at $48 \mathrm{~h}$ following treatment with JA and ET, respectively, although their induction levels were lower than those with SA. OsWRKY62 and 76 are negative regulators of disease resistance responses in rice $[25,26]$, and no Brachypodium homologs for OsWRKY62 were found, whereas three genes, Bradi4g30360, Bradi1g30870 and Bradi3g06070, showed similarity to OsWRKY76. In the RNA-seq results

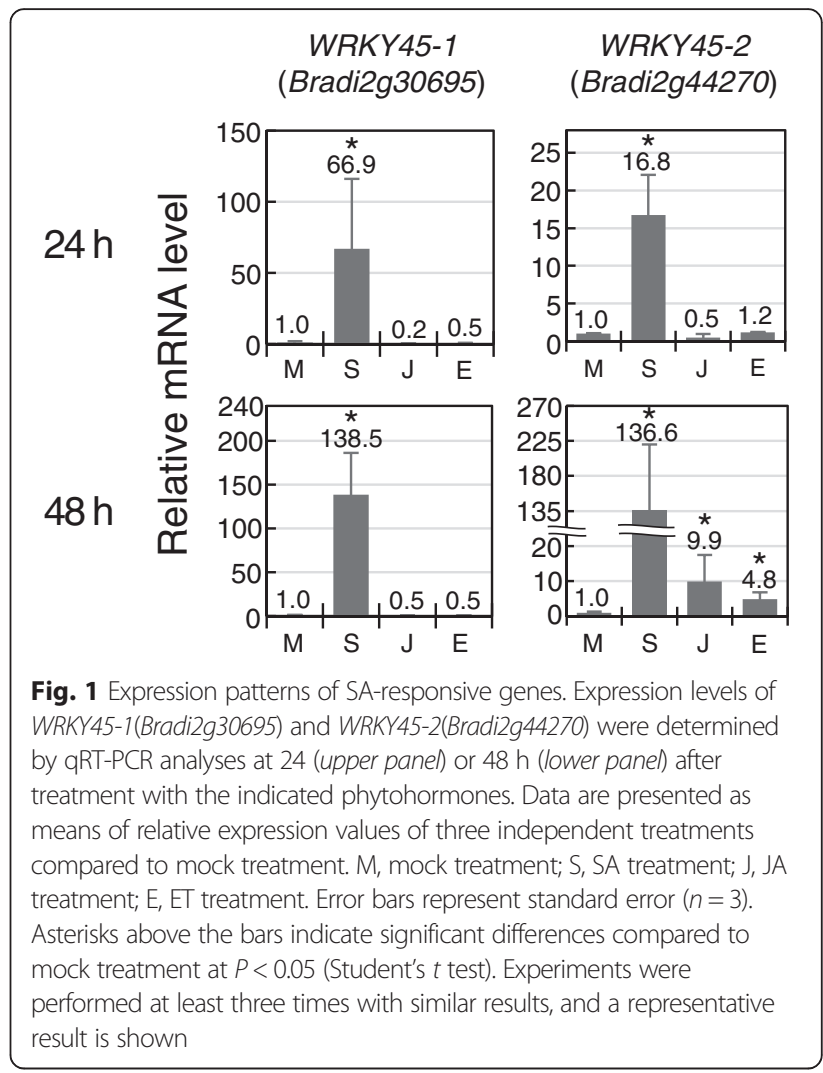

by Kakei et al., only Bradi4g30360, the gene most similar to OsWRKY76 among the Brachypodium homologs, was induced (with a $\log _{2}$ ratio of 3 ) at $3 \mathrm{~h}$ after SA treatment.

During disease resistance response in Arabidopsis, SA is biologically synthesized to induce defence responses and is subsequently metabolised to reset the immunity mode. One of the major SA metabolism pathways is glycosylation, in which SA glucosyltransferase (SAGT) conjugates a glucose moiety to SA to produce SA-O- $\beta$-Dglucoside (SAG) using UDP-glucose as a donor. SAG is an inactive form of SA [28]. In Arabidopsis and rice, SA treatment leads to increased expression of $S A G T$ genes $[29,30]$. Under the hypothesis that $S A G T$ is an SA marker, Brachypodium SAGT genes were retrieved from the cDNA database. Four and three Brachypodium homologs of Arabidopsis UGT76B1 and UGT74F1, respectively, showing identities of $>40 \%$ in their amino acid sequences, were identified. One homolog with the highest similarity to OsSGT1 was also selected. In Brachypodium, no induction by SA was detected for these 7 SAGT genes (Table 2). Instead, we found that Bradi4g41410 was induced by ET (Fig. 3). It is not clear whether the genes used in this study function as SAGT, given that more than 170 predicted UGT genes were found in the Brachypodium genome and sequence similarity using whole length does not always reflect 
functional identity. Other studies are needed to identify the players involved in SA metabolism in Brachypodium.

Allene oxide synthase (AOS) and lipoxygenase (LOX) are required for JA biosynthesis [31]. Positive feedback regulation in transcription of these enzyme-encoding genes by JA is well understood and they are used as JA markers in various plant species. In Arabidopsis, expression of AtAOS 2 and AtLOX2 were upregulated by JA [32]. In rice, induction of OsAOS2 and OsLOX1 was detected at $6 \mathrm{~h}$ after JA treatment, according to the rice global expression profile database RiceXPro [33]. In barley, JA responsiveness of AOS (contig3096_s_at) and LOX (contig2306_s_at) was validated by microarray analysis and semi-quantitative RT-PCR [34]. Four Brachypodium genes, Bradi1g69330, Bradi1g07480, Bradi3g08160 and Bradi3g01110, were identified as homologs of OsAOS2 by blastp search, and Bradilg69330, with the highest score, was used in this study. Its deduced protein sequence also shows high similarity to barley AOS. We detected strong induction of this Brachypodium AOS gene at $24 \mathrm{~h}$ after JA treatment, and its level was doubled at the $48 \mathrm{~h}$ time point (Fig. 2). For $L O X, 10$ genes (Bradi1g11670, Bradilg11680, Bradilg09260, Bradi1g09270, Bradi3g59710, Bradi5g11590, Bradi1g72690, Bradi3g39980, Bradi3g07010 and Bradi3g07000) were found as OsLOX1 (Os03g0700700) homologs. The most similar Bradilg11670 gene has been shown to be expressed after infection by the fungal pathogen Sclerotinia homeocarpa in the resistant Brachypodium accession 208126 [35]. We accordingly checked its response to JA. As shown in Fig. 2, 3.0- and 4.7-fold expression changes were observed at 24 and $48 \mathrm{~h}$, respectively, after hormone treatment. These results suggest that both genes would be useful JA markers.
During the disease resistance response, plants use phenylpropanoid compounds for the biosynthesis of lignin, flavonoids, and phytoalexins, which are required for the fortification of cell walls and production of antimicrobials [36]. 4-Coumarate:CoA ligase (4CL) and phenylalanine ammonia lyase (PAL) are key enzymes in this metabolic pathway, and the transcriptional upregulation of $P A L$ and $4 C L$ after elicitor treatment and pathogen inoculation have been reported in Arabidopsis, rice and Brachypodium [35, 37-39]. In Brachypodium, three 4CL homologs, Bradi3g37300, Bradi3g05750 and Bradi1g31320, were identified by blastp search using the protein sequence of Arabidopsis At1g51680 as a query (E value $=0$ ). Similarly, Bradi5g15830, Bradi3g48840, Bradi3g49280, Bradi3g49260, Bradi3g49270, Bradi3g47110, Bradi3g47120 and Bradi3g49250 were found as homologs of AtPAL1 (At2g37040). Bradi3g37300 as a representative of $4 C L$ and Bradi3g48840 for PAL were markedly induced at $24 \mathrm{~h}$ after JA treatment, with further-increased levels at $48 \mathrm{~h}$ (Fig. 2). We checked the expression of rice OsPAL1 and Os4CL5 using the RiceXPro database [33] and found that they were also induced within $6 \mathrm{~h}$ after JA treatment, in accord with our result. In our study, expression of Brachypodium $4 C L$ was also detected by both SA and ET at $48 \mathrm{~h}$. These Brachypodium $4 C L$ and $P A L$ genes have also been reported to be induced by JA ( $\log _{2}$ ratio $=1.59$ and 1.96 , respectively) $1 \mathrm{~h}$ after $30 \mu \mathrm{M}$ MeJA treatment [20].

Tryptophan aminotransferase of Arabidopsis 1 (TAA1)-related (TAR) is required for the biosynthesis of indole-3-pyruvic acid from L-tryptophan in Arabidopsis [40] and its expression is upregulated by ET [41]. In Brachypodium, the expression levels of two TAR homologs, BdTARL1 (Bradi2g34400) and BdTARL2 (Bradi2g04290),

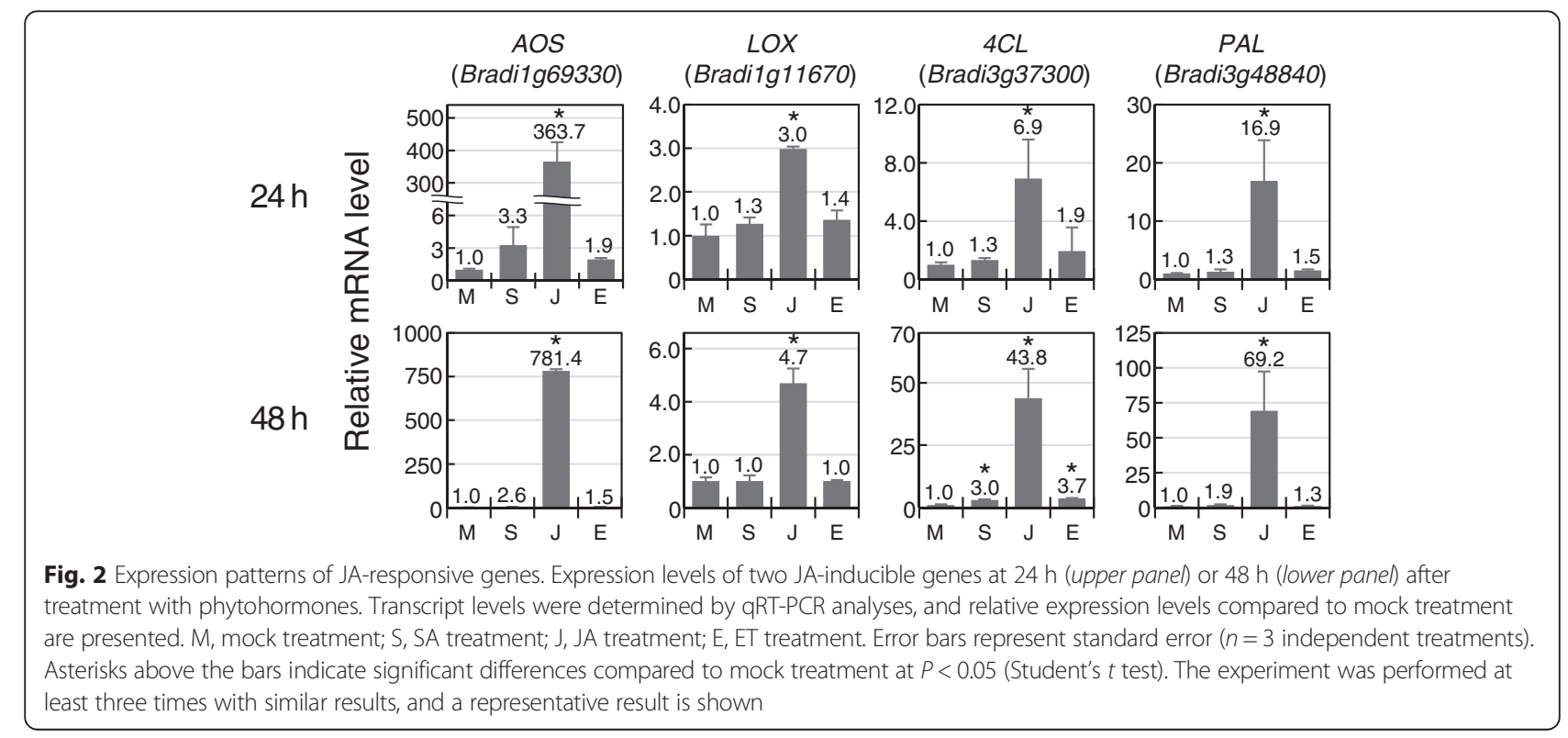


have been shown to be increased at $3 \mathrm{~h}$ after ACC treatment (Table 2) [21]. Under our experimental conditions, transcription of BdTARL2 but not BdTARL1 was significantly induced at both 24 and $48 \mathrm{~h}$ after ethephon treatment (Fig. 3). BdTARL2 may have been expressed continuously by ET from 3 to $48 \mathrm{~h}$ after the treatment. Because genes involved in biosynthesis and signalling of ET are often transcriptionally activated by ET in Arabidopsis, we selected ACS (ACC SYNTHASE) (Bradi1g49966), ERF (ETHYLENE RESPONSIVE FACTOR) (Bradi2g52370) and EIN3 (ETHYLENE-INSENSITIVE3) (Bradi1g63780) as candidate ET-responsive genes. They were the closest homologs to the corresponding rice genes (Table 1) [42-44]. In our study, their transcription did not respond to ET (Table 2). In Brachypodium, we found a single homolog of EIN3, but there were 4 ACS homologs and over 100 homologs of $A P 2 / E R F$ family genes. Thus, it is still possible that there are ETresponsive $A C S$ and $E R F$ in the genome. RNA-seq analysis at $3 \mathrm{~h}$ after ACC treatment identified only an EIN4 homolog (Bradi5g00700) as an ET-responsive gene [20].

In rice, pathogenesis-related genes PR5 and PR10 (PBZ1; PROBENAZOLE-INDUCED PROTEIN1) are induced by ET or chitin, typical PAMPs $[45,46]$. They belong to multigene families in rice, and we found 32 and 5 homologs in Brachypodium for PR5 and PR10, respectively. The expression levels of Bradilg33540 and

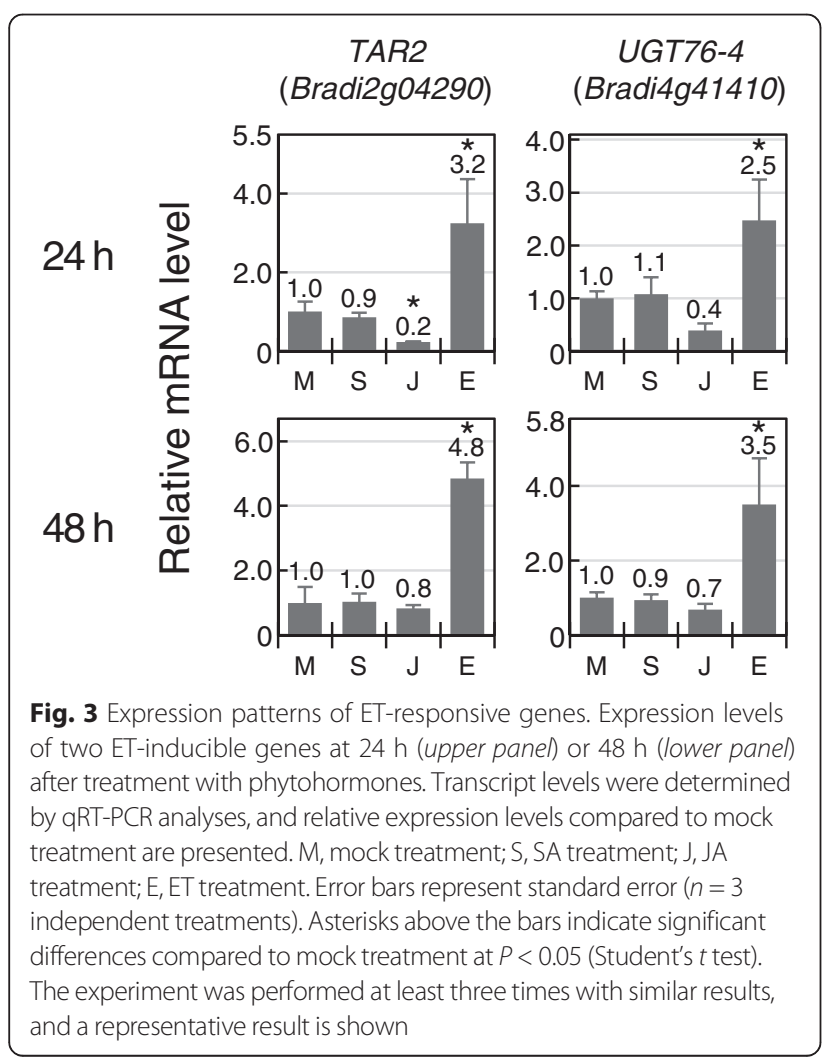

Bradi4g05040 as marker candidates for PR5 and PR10, respectively, were evaluated because they are the homologs most similar to OsPR5 and OsPR10, and Bradi1g33540 has already been shown to be induced by pathogens [19]. However, no induction by phytohormone treatment could be detected under our conditions (Table 2).

In summary, we successfully identified 2, 4 and 2 marker genes for SA, JA and ET, respectively. They may be useful tools for the characterisation of defence responses induced in Brachypodium in various hostparasite interactions.

\section{Characterisation of the phytohormone responsiveness of the BdPR1 gene family in Brachypodium}

SA is used for plant defence mainly against biotrophic pathogens, and JA and ET are mainly used against necrotrophic pathogens [47]. In Arabidopsis, SA and JA exert an antagonistic effect on each other [48]. For instance, SA treatment suppresses JA-inducible genes such as PDF1.2, VSP1, LOX2, AOS, AOC2 and OPR3 [49]. Recently, a genome-wide transcriptional analysis in rice using microarray revealed that more than half of 313 genes upregulated by benzothiadiazole (BTH), a functional analogue of SA, are also induced by JA, although a third of them were suppressed by JA [50]. This gene set, positively regulated by both SA and JA, is defined as a common defence system that is possibly used in response to various biotic and abiotic stresses in rice [50, 51]. OsWRKY45 and several OsPR1 genes are examples of genes belonging to this group with their expression levels increased by both SA and JA [52, 53].

On the other hand, this common defence system is not found in tobacco and Arabidopsis. In tobacco, PR1family proteins consist of acidic and basic groups regulated by SA and JA, respectively, and the induction of each gene was antagonistically suppressed by the other hormones [54]. In Arabidopsis, only AtPR1 (At2g14610) among 22 PR1-family genes is responsive to SA and pathogen inoculation based on microarray data [55], although AtPRB1 was shown to be weakly induced by MeJA and ET in root [56]. These situations may depend on differences between rice and dicots in the SA signalling cascade [57]. We accordingly speculate that this common defence system is a characteristic feature of monocots. However, rice contains a high level of endogenous SA under normal conditions, unlike other monocots such as barley and Brachypodium [6, 58]. To determine whether this common defence system is specific to rice and arose during domestication or is shared by all monocots, we characterised the response nature of PR1-family genes in Brachypodium and compared it with those of rice and Arabidopsis. 
A blastp search of the protein sequence of AtPR1 against the database of RIKEN Brachypodium FLcDNA clones, to identify Brachypodium PR1 homologs, yielded 11 genes, defined as the $B d P R 1$ family, with high similarities in their deduced protein sequences (E value $<1 \mathrm{E}-10$ ). Among them, 5 and 4 genes were located on chromosomes 1 and 3, respectively, and the remaining 2 genes were found on chromosomes 2 and 4. According to rice PR1 gene nomenclature [52], these $B d P R 1$ genes were also designated based on their chromosomal locations. The order of precedence depends on both chromosome number and position from the $5^{\prime}$ end. For example, the 5 BdPR1 members on chromosome 1 were named BdPR1-1, BdPR1-2, BdPR1-3, BdPR1-4 and BdPR1-5 in order from $5^{\prime}$ to $3^{\prime}$. The gene on chromosome 2 was named BdPR1-6.
We designed primers for specific detection of each $B d P R 1$ gene in qRT-PCR experiments and evaluated their expressions at 24 and $48 \mathrm{~h}$ after treatment with SA, JA, or ET (Fig. 4). According to their expression patterns, $B d P R 1$ members were classified into three groups. Group A contains five BdPR1 genes whose transcriptions were not upregulated by any phytohormone (Fig. 4a). Instead, their expressions were significantly or likely suppressed at 24 or $48 \mathrm{~h}$ after treatment with these phytohormones. Such suppression was similarly observed for BdPR1-1, BdPR1-6 and BdPR1-8, which are categorised into other groups, at $24 \mathrm{~h}$ after phytohormone treatment. Two genes were in group B, members of which were responsive to only a single phytohormone, JA (Fig. 4b). BdPR1-2 was induced at both 24 and $48 \mathrm{~h}$, whereas BdPR1-6 was upregulated only at $48 \mathrm{~h}$. Group $\mathrm{C}$ comprises 4 genes induced by more than two

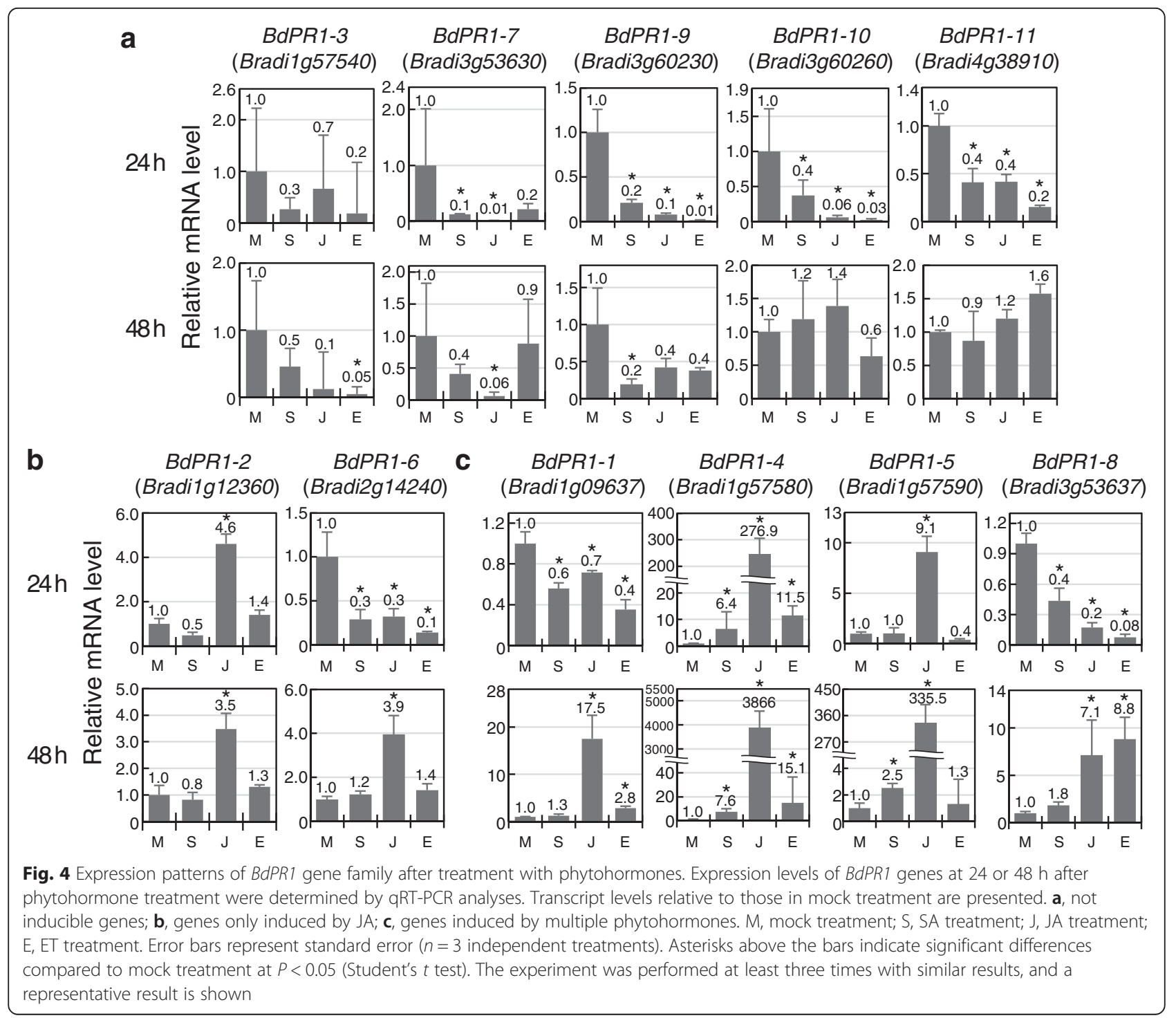


phytohormones (Fig. 4c). Transcription of BdPR1-1 and $B d P R 1-8$ was induced by JA and ET at $48 \mathrm{~h}$ after treatment. BdPR1-5 expression responded to JA at $24 \mathrm{~h}$ and its level was further increased at $48 \mathrm{~h}$. A weak response of this gene to SA was also detected at $48 \mathrm{~h}$. As for $B d P R 1-4$, its transcription was induced by all of the tested phytohormones. Its induction was especially sensitive to JA, and massive transcription was detected at $48 \mathrm{~h}$.

Our results revealed that some of the Brachypodium $P R 1$ genes were induced by multiple phytohormones, as reported in rice [52]. Using the predicted protein sequences of 11, 12 and 22 PR1 families of Brachypodium, rice and Arabidopsis, respectively, a phylogenetic tree was constructed by the UPGMA (Unweighted Pair Group Method with Arithmetic mean) method (Fig. 5). Protein sequences of the rice OsPR1 and the Arabidopsis AtPR1 family were obtained from the MSU Rice Genome Annotation Project and the Arabidopsis Information Resource (TAIR), respectively. The resulting tree illustrates that Brachypodium and rice contain similar sets of PR1 family genes apart from Arabidopsis, and it

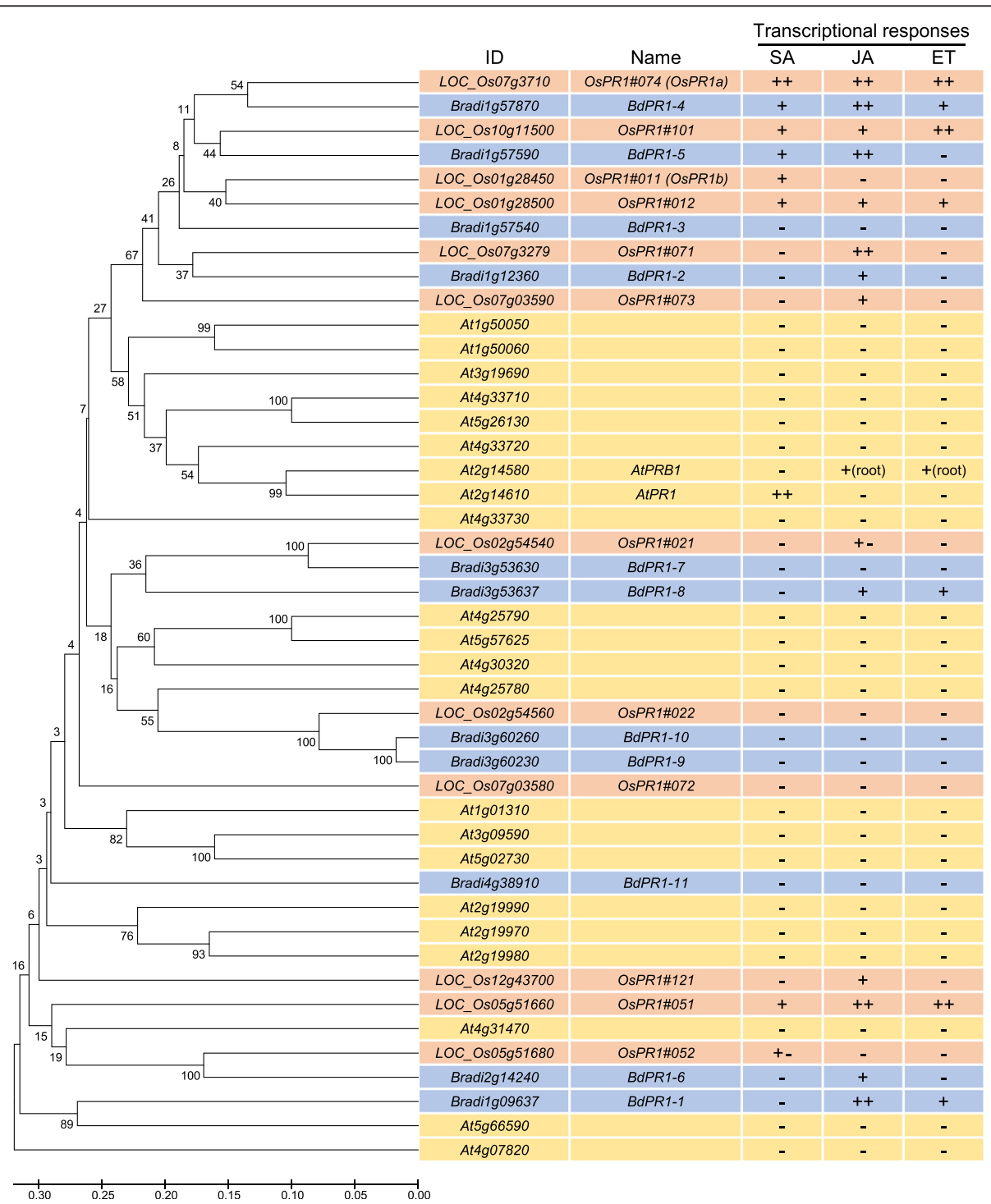

Fig. 5 Phylogenetic analysis of PR1 gene families in Arabidopsis, rice and Brachypodium. A phylogenetic tree of PR1 gene families of Arabidopsis, rice and Brachypodium was constructed with MEGA software (http://www.megasoftware.net/) using the UPGMA method with bootstrap values (1000). Phytohormone inducibilities of BdPR1 family analysed in this study and those of the AtPR1 family and OsPR1 family reported in van Loon et al. (2006) and Mitsuhara et al. (2008), respectively are summarised in the right column [52, 55]. Induction status is presented as follows: ++, significantly induced more than 10 -fold compared to the mock treatment; +, significantly induced more than 2 -fold compared to the mock treatment; -, not inducible; +-, gene whose induction or expression was not clear 
suggests the difference between monocots and dicots in constitution of $P R 1$ family proteins. In the right columns of Fig. 5, we summarise the phytohormone responsiveness of these Brachypodium PR1 genes as revealed in this study and the reported information for rice OsPR1 and Arabidopsis AtPR1 genes. In AtPR1 genes, only two genes (At4g25780, At5g66590) were classified into the same clade of monocot PR1 genes, whereas remaining 20 genes, which contained phytohormone responsive AtPR1 and AtPRB1, formed independent clades. Some of the PR1 genes from Brachypodium and rice classified into the same clade showed similar expression response patterns to the phytohormones. For example, BdPR1-4 and OsPR1\#074 (OsPR1a) or BdPR1-5 and OsPR1\#101 responded to multiple phytohormones, whereas $B d P R 1$ 7, BdPR1-9, BdPR1-10, OsPR1\#021 and OsPR1\#022 were not induced by any phytohormones. BdPR1-2 and OsPR1\#071 were induced by only JA. Other gene pairs showed different expression patterns, suggesting different roles of the PR1 family between these plant species.

From these situations, we hypothesized that a common defence system is present in Brachypodium and that the system is conserved among monocot plants. This idea is also supported by our findings that at least WRKY45-2, 4CL, BdPR1-4 and BdPR1-5 were regulated by both SA and JA (Figs. 1, 2 and 4c). A comprehensive transcriptome analysis of Brachypodium using RNA-seq or microarrays may confirm this hypothesis.

\section{Conclusions}

Genome deciphering by next-generation sequencing and comprehensive transcriptome analysis with RNAseq enable comparative genomics in many crop species. Distinctive features in crops often impede the progress of detailed molecular analysis, but a large picture of plant immunity is available only in Arabidopsis and rice at present. Given that Brachypodium has attractive advantages that can overcome the limitations of crop research especially for Pooideae crops attributed to slow growth speed, large genome size, high ploidy and so on, it is expected to provide knowledge bearing on the commonality or uniqueness of defence systems among plant species. In this study, we identified the phytohormone marker genes WRKY45-1 and WRKY45-2 for SA; AOS, LOX, 4CL, PAL, PR1-2, PR1-5 and PR1-6 for JA and TAR and UGT76-4 for ET (Figs. 1, 2, 3 and 4). Having been selected for responsiveness on the bases of both time point and intensity, which are parameters used for monitoring plant reactions during infection by many phytopathogens, these genes should be useful tools not only for describing spatiotemporal immune responses to specific pathogens in Brachypodium but also for comparing them with those to other pathogens in a unified framework. The comparison of expression profiles of $P R 1$ family genes suggests that Brachypodium has phytohormone responses more similar to those of rice than of Arabidopsis.

\section{Methods}

Plant materials and growth conditions

The Brachypodium distachyon cultivar $\mathrm{Bd} 21$ was used. Brachypodium seeds were germinated on moist filter paper. After 7 days, the seedlings were transferred to wells of 24-well microtiter plates filled with soil and grown in a growth chamber (LPH-350S; Nippon Medical \& Chemical Instruments, Osaka, Japan) at $23{ }^{\circ} \mathrm{C}$ under a $20 \mathrm{~h}$ light/4 h dark photoperiod [13].

\section{Phytohormone treatment}

Sodium salicylate (SA; Wako, Osaka, Japan), MeJA (JA; Wako, Osaka, Japan) and ethephon (Sigma-Aldrich, St. Louis, MO, USA), an ET generator, were used as phytohormones. Whole Bd21 seedlings grown for 3 to 4 weeks were immersed in water (mock treatment) or a plant hormone solution ( $1 \mathrm{mM} \mathrm{SA}, 100 \mu \mathrm{l} \mathrm{MeJA}$, or $100 \mu \mathrm{M}$ ethephon) using 50-mL conical tubes. The seedlings were incubated for 24 or $48 \mathrm{~h}$ at $23{ }^{\circ} \mathrm{C}$ under a $20 \mathrm{~h}$ light $/ 4 \mathrm{~h}$ dark photoperiod. Then, the first and second fully expanded leaves from the top of the seedlings were collected in 2-mL tubes and frozen in liquid nitrogen.

\section{RNA extraction and gene expression analysis}

The frozen samples were crushed with four zirconia beads ( $\varnothing 2 \mathrm{~mm}$ ) using a Shake Master Neo (BMS, Tokyo, Japan). Total RNA was extracted with a Total RNA Purification Kit (JenaBioscience, Jena, Germany) with oncolumn DNase treatment (Invitrogen, Carlsbad, CA, USA). RNA concentration and purity were validated with a DS-11 spectrophotometer (Denovix, Wilmington, DE, USA). cDNA was synthesized from each sample with the PrimeScript RT reagent kit with gDNA Eraser (Takara, Shiga, Japan). Gene expression analyses were performed by qRT-PCR using a KAPA SYBR Fast qPCR Kit (KAPA BIOSYSTEMS, Woburn, MA, USA) with a GVP-9600 real-time PCR instrument (Shimadzu, Kyoto, Japan). The quantification of target transcripts was performed using the GVP-9600 internal software GVP gene detection system, and the data were normalised to the BdUbi4 gene (Bradi3g04730), which has been established as a reference gene for expression studies in $B$. distachyon [59]. Primers used in this study are listed in Additional file 2: Table S1.

\section{Availability of data and materials}

All supporting data can be found within the manuscript and its additional files. 


\section{Additional files}

Additional file 1: Figure S1. Protein sequence alignments of OsWRKY45, BdWRKY45-1 and BdWRKY45-2 (PPTX 145 kb)

Additional file 2: Table S1. Primers used in this study (DOCX $32 \mathrm{~kb}$ )

\section{Abbreviations}

ACC: 1-aminocyclopropane-1-carboxylic acid; ACS: ACC synthase; AOC: allene oxide cyclase; AOS: allene oxide synthase; BTH: benzothiadiazole; CoA: coenzyme A; EIN: ethylene insensitive; ERF: ethylene responsive factor; ET: ethylene; ETI: effector-triggered immunity; FLCDNA: full-length CDNA; JA: jasmonic acid; LOX: lipoxygenase; MeJA: mehyl jasmonate; NLR: cytoplasmic nucleotide-binding domain and leucine-rich repeat: OPR: 12-oxo-phytodienoic acid reductase; PAL: phenylalanine ammonia lyase; PAMPS/MAMPs: pathogen- or microbe- associated molecular patterns; PBZ1: probenazole-induced protein 1; PDF: plant defensin; PR: pathogenesisrelated; PTI/MTI: PAMPS/MAMPs-triggered immunity; qRT-PCR: quantitative reverse-transcription polymerase chain reaction; SA: salicylic acid; SAG: SA-O$\beta$-D-glucoside; SAGT: SA glucosyltransferase; TAR: tryptophan aminotransferase of arabidopsis 1 (TAA1)-related; UPGMA: unweighted pair group method with arithmetic mean; VSP: vegetative storage protein.

\section{Competing interests}

The authors declare that they have no competing interests.

\section{Authors' contributions}

YK, KM, HM, MY, YI, KT and YN conceived of the study and designed the experiments. YK, MK, YY, MW and YN carried out the experiments and performed the statistical analysis. $Y K, Y O$ and $Y N$ drafted the manuscript. $Y O$, $\mathrm{KM}, \mathrm{HM}, \mathrm{MY}, \mathrm{YI}$ and $\mathrm{KT}$ contributed to analysis and interpretation of data and the critical revision of the manuscript. All authors read and approved the final manuscript.

\section{Acknowledgements}

This research was supported by ALCA (Advanced Low Carbon Technology Research and Development Program) Grant to YN from the Japan Science and Technology Agency, KAKENHI Grant 25292035 to YN from the Ministry of Education, Culture, Sports, Science and Technology of Japan and a grant to YN from the Japan Foundation for Applied Enzymology.

\section{Author details}

${ }^{1}$ Graduate School of Environmental and Life Science, Okayama University, Kita-ku, Okayama, Japan. ${ }^{2}$ Cellulose Production Research Team, Biomass Engineering Research Division, RIKEN Center for Sustainable Resource Science, Tsurumi, Yokohama, Japan.

\section{Received: 27 December 2015 Accepted: 26 February 2016}

\section{Published online: 02 March 2016}

\section{References}

1. Boller T, Felix G. A renaissance of elicitors: perception of microbe-associated molecular patterns and danger signals by pattern-recognition receptors. Annu Rev Plant Biol. 2009;60:379-406.

2. Jones JD, Dangl JL. The plant immune system. Nature. 2006;444(7117): 323-9.

3. Mersmann S, Bourdais G, Rietz S, Robatzek S. Ethylene signaling regulates accumulation of the FLS2 receptor and is required for the oxidative burst contributing to plant immunity. Plant Physiol. 2010;154(1):391-400.

4. Bari $\mathrm{R}$, Jones JD. Role of plant hormones in plant defence responses. Plant Mol Biol. 2009;69(4):473-88

5. Silverman P, Seskar M, Kanter D, Schweizer P, Metraux JP, Raskin I. Salicylic acid in rice (Biosynthesis, conjugation, and possible role). Plant Physiol. 1995; 108(2):633-9.

6. Huckelhoven R, Fodor J, Preis C, Kogel KH. Hypersensitive cell death and papilla formation in barley attacked by the powdery mildew fungus are associated with hydrogen peroxide but not with salicylic acid accumulation. Plant Physiol. 1999;119(4):1251-60.

7. Iwai T, Seo S, Mitsuhara I, Ohashi Y. Probenazole-induced accumulation of salicylic acid confers resistance to Magnaporthe grisea in adult rice plants. Plant Cell Physiol. 2007;48(7):915-24.
8. Ward ER, Uknes SJ, Williams SC, Dincher SS, Wiederhold DL, Alexander DC, et al. Coordinate gene activity in response to agents that induce systemic acquired resistance. Plant Cell. 1991;3(10):1085-94.

9. Lyons R, Manners JM, Kazan K. Jasmonate biosynthesis and signaling in monocots: a comparative overview. Plant Cell Rep. 2013;32(6): 815-27.

10. Zhu F, Xi DH, Yuan S, Xu F, Zhang DW, Lin HH. Salicylic acid and jasmonic acid are essential for systemic resistance against tobacco mosaic virus in Nicotiana benthamiana. Mol Plant Microbe Interact. 2014;27(6):567-77.

11. Brkljacic J, Grotewold E, Scholl R, Mockler T, Garvin DF, Vain P, et al. Brachypodium as a model for the grasses: today and the future. Plant Physiol. 2011;157(1):3-13

12. Initiative IB. Genome sequencing and analysis of the model grass Brachypodium distachyon. Nature. 2010;463(7282):763-8.

13. Mochida K, Uehara-Yamaguchi Y, Takahashi F, Yoshida T, Sakurai T, Shinozaki K. Large-scale collection and analysis of full-length cDNAs from Brachypodium distachyon and integration with Pooideae sequence resources. PLoS One. 2013;8(10):e75265.

14. Onda Y, Hashimoto K, Yoshida T, Sakurai T, Sawada Y, Hirai MY, et al. Determination of growth stages and metabolic profiles in Brachypodium distachyon for comparison of developmental context with Triticeae crops. Proc Biol Sci. 2015;282(1811):20150964. doi:10.1098/rspb.2015.0964.

15. Fitzgerald TL, Powell JJ, Schneebeli K, Hsia MM, Gardiner DM, Bragg JN, et al. Brachypodium as an emerging model for cereal-pathogen interactions. Ann Bot. 2015;115(5):717-31.

16. Peraldi A, Beccari G, Steed A, Nicholson P. Brachypodium distachyon: a new pathosystem to study Fusarium head blight and other Fusarium diseases of wheat. BMC Plant Biol. 2011:11:100

17. Parker D, Beckmann M, Enot DP, Overy DP, Rios ZC, Gilbert M, et al. Rice blast infection of Brachypodium distachyon as a model system to study dynamic host/pathogen interactions. Nat Protoc. 2008;3(3):435-45.

18. Mandadi KK, Scholthof KB. Characterization of a viral synergism in the monocot Brachypodium distachyon reveals distinctly altered host molecular processes associated with disease. Plant Physiol. 2012;160(3):1432-52.

19. Pogorelko G, Lionetti V, Fursova O, Sundaram RM, Qi M, Whitham SA, et al. Arabidopsis and Brachypodium distachyon transgenic plants expressing Aspergillus nidulans acetylesterases have decreased degree of polysaccharide acetylation and increased resistance to pathogens. Plant Physiol. 2013; 162(1):9-23.

20. Kakei Y, Mochida K, Sakurai T, Yoshida T, Shinozaki K, Shimada Y. Transcriptome analysis of hormone-induced gene expression in Brachypodium distachyon. Sci Rep. 2015;5:14476.

21. Pacheco-Villalobos D, Sankar M, Ljung K, Hardtke CS. Disturbed local auxin homeostasis enhances cellular anisotropy and reveals alternative wiring of auxin-ethylene crosstalk in Brachypodium distachyon seminal roots. PLoS Genet. 2013;9(6):e1003564.

22. Altschul SF, Gish W, Miller W, Myers EW, Lipman DJ. Basic local alignment search tool. J Mol Biol. 1990;215(3):403-10.

23. Rozen S, Skaletsky H. Primer3 on the WWW for general users and for biologist programmers. Methods Mol Biol. 2000;132:365-86.

24. Shimono M, Sugano S, Nakayama A, Jiang CJ, Ono K, Toki S, et al. Rice WRKY45 plays a crucial role in benzothiadiazole-inducible blast resistance. Plant Cell. 2007;19(6):2064-76.

25. Peng Y, Bartley LE, Chen X, Dardick C, Chern M, Ruan R, et al. OsWRKY62 is a negative regulator of basal and $X a 21$-mediated defense against Xanthomonas oryzae pv. oryzae in rice. Mol Plant. 2008;1(3):446-58.

26. Yokotani N, Sato Y, Tanabe S, Chujo T, Shimizu T, Okada K, et al. WRKY76 is a rice transcriptional repressor playing opposite roles in blast disease resistance and cold stress tolerance. J Exp Bot. 2013:64(16):5085-97.

27. Kawahara $Y$, Oono $Y$, Kanamori H, Matsumoto $T$, Itoh $T$, Minami E. Simultaneous RNA-seq analysis of a mixed transcriptome of rice and blast fungus interaction. PLoS One. 2012;7:e49423.

28. Dempsey DA, Vlot AC, Wildermuth MC, Klessig DF. Salicylic acid biosynthesis and metabolism. Arabidopsis Book. 2011;9:e0156.

29. Umemura K, Satou J, Iwata M, Uozumi N, Koga J, Kawano T, et al. Contribution of salicylic acid glucosyltransferase, OSSGT1, to chemically induced disease resistance in rice plants. Plant J. 2009;57(3):463-72.

30. Noutoshi Y, Okazaki M, Kida T, Nishina Y, Morishita Y, Ogawa T, et al. Novel plant immune-priming compounds identified via high-throughput chemical screening target salicylic acid glucosyltransferases in Arabidopsis. Plant Cell. 2012;24(9):3795-804. 
31. Mueller MJ. Enzymes involved in jasmonic acid biosynthesis. Physiol Plant. 1997;100:653-63.

32. Sasaki Y, Asamizu E, Shibata D, Nakamura Y, Kaneko T, Awai K, et al. Monitoring of methyl jasmonate-responsive genes in Arabidopsis by cDNA macroarray: self-activation of jasmonic acid biosynthesis and crosstalk with other phytohormone signaling pathways. DNA Res. 2001;8(4):153-61.

33. Sato $Y$, Takehisa $H$, Kamatsuki K, Minami H, Namiki N, Ikawa H, et al. RiceXPro version 3.0: expanding the informatics resource for rice transcriptome. Nucleic Acids Res. 2013;41(Database issue):D1206-13.

34. Walia H, Wilson C, Condamine P, Liu X, Ismail AM, Close TJ. Large-scale expression profiling and physiological characterization of jasmonic acidmediated adaptation of barley to salinity stress. Plant Cell Environ. 2007; 30(4):410-21.

35. Sandoya GV, Buanafina MMO. Differential responses of Brachypodium distachyon genotypes to insect and fungal pathogens. Physiol Mol Plant Pathol. 2014:85:53-64.

36. Dixon RA, Achnine L, Kota P, Liu CJ, Reddy MS, Wang L. The phenylpropanoid pathway and plant defence-a genomics perspective. Mol Plant Pathol. 2002;3(5):371-90.

37. Tonnessen BW, Manosalva P, Lang JM, Baraoidan M, Bordeos A, Mauleon R, et al. Rice phenylalanine ammonia-lyase gene OsPAL4 is associated with broad spectrum disease resistance. Plant Mol Biol. 2015;87(3):273-86.

38. Truman W, Bennett MH, Kubigsteltig I, Turnbull C, Grant M. Arabidopsis systemic immunity uses conserved defense signaling pathways and is mediated by jasmonates. Proc Natl Acad Sci U S A. 2007;104(3):1075-80.

39. Davis KR, Ausubel FM. Characterization of elicitor-induced defense responses in suspension-cultured cells of Arabidopsis. Mol Plant Microbe Interact. 1989;2(6):363-8.

40. Mano Y, Nemoto K. The pathway of auxin biosynthesis in plants. J Exp Bot. 2012;63(8):2853-72.

41. Stepanova AN, Robertson-Hoyt J, Yun J, Benavente LM, Xie DY, Dolezal K, et al. TAA1-mediated auxin biosynthesis is essential for hormone crosstalk and plant development. Cell. 2008;133(1):177-91.

42. Mao C, Wang S, Jia Q, Wu P. OsEIL 1, a rice homolog of the Arabidopsis EIN3 regulates the ethylene response as a positive component. Plant Mol Biol. 2006;61(1-2):141-52

43. Hu Y, Zhao L, Chong K, Wang T. Overexpression of OsERF1, a novel rice ERF gene, up-regulates ethylene-responsive genes expression besides affects growth and development in Arabidopsis. J Plant Physiol. 2008;165(16): 1717-25.

44. Iwai T, Miyasaka A, Seo S, Ohashi Y. Contribution of ethylene biosynthesis for resistance to blast fungus infection in young rice plants. Plant Physiol. 2006;142(3):1202-15.

45. Agrawal GK, Rakwal R, Jwa NS. Differential induction of three pathogenesisrelated genes, PR10, PR1b and PR5 by the ethylene generator ethephon under light and dark in rice (Oryza sativa L.) seedlings. J Plant Physiol. 2001; 158(1):133-7.

46. Kouzai Y, Mochizuki S, Nakajima K, Desaki Y, Hayafune M, Miyazaki H, et al. Targeted gene disruption of OSCERK1 reveals its indispensable role in chitin perception and involvement in the peptidoglycan response and immunity in rice. Mol Plant Microbe Interact. 2014;27(9):975-82.

47. Glazebrook J. Contrasting mechanisms of defense against biotrophic and necrotrophic pathogens. Annu Rev Phytopathol. 2005:43:205-27.

48. Kunkel BN, Brooks DM. Cross talk between signaling pathways in pathogen defense. Curr Opin Plant Biol. 2002;5(4):325-31.

49. Leon-Reyes A, Van der Does D, De Lange ES, Delker C, Wasternack C, Van Wees SC, et al. Salicylate-mediated suppression of jasmonate-responsive gene expression in Arabidopsis is targeted downstream of the jasmonate biosynthesis pathway. Planta. 2010;232(6):1423-32.

50. Tamaoki D, Seo S, Yamada S, Kano A, Miyamoto A, Shishido H, et al. Jasmonic acid and salicylic acid activate a common defense system in rice. Plant Sig Behav. 2013;8(6):e24260.

51. De Vleesschauwer D, Gheysen G, Höfte M. Hormone defense networking in rice: tales from a different world. Trends Plant Sci. 2013;18(10):555-65.

52. Mitsuhara I, Iwai T, Seo S, Yanagawa Y, Kawahigasi H, Hirose S, et al. Characteristic expression of twelve rice $P R 1$ family genes in response to pathogen infection, wounding, and defense-related signal compounds (121/180). Mol Gen Genom. 2008;279(4):415-27.

53. Yamada S, Kano A, Tamaoki D, Miyamoto A, Shishido H, Miyoshi S, et al. Involvement of OsJAZ8 in jasmonate-induced resistance to bacterial blight in rice. Plant Cell Physiol. 2012;53(12):2060-72.
54. Niki T, Mitsuhara I, Seo S, Ohtsubo N, Ohashi Y. Antagonistic Effect of salicylic acid and jasmonic acid on the expression of pathogenesis-related (PR) protein genes in wounded mature tobacco leaves. Plant Cell Physiol. 1998;39(5):500-7.

55. van Loon LC, Rep M, Pieterse CM. Significance of inducible defense-related proteins in infected plants. Annu Rev Phytopathol. 2006;44:135-62.

56. Santamaria M, Thomson CJ, Read ND, Loake GJ. The promoter of a basic PR1-like gene, AtPRB1, from Arabidopsis establishes an organ-specific expression pattern and responsiveness to ethylene and methyl jasmonate. Plant Mol Biol. 2001;47(5):641-52.

57. Takatsuji H. Development of disease-resistant rice using regulatory components of induced disease resistance. Front Plant Sci. 2014;5:630.

58. Ayliffe M, Singh D, Park R, Moscou M, Pryor T. Infection of Brachypodium distachyon with selected grass rust pathogens. Mol Plant Microbe Interact. 2013:26(8):946-57.

59. Chambers JP, Behpouri A, Bird A, Ng CK. Evaluation of the use of the polyubiquitin genes, Ubi4 and Ubi10 as reference genes for expression studies in Brachypodium distachyon. PLoS One. 2012;7:e49372.

\section{Submit your next manuscript to BioMed Central and we will help you at every step:}

- We accept pre-submission inquiries

- Our selector tool helps you to find the most relevant journal

- We provide round the clock customer support

- Convenient online submission

- Thorough peer review

- Inclusion in PubMed and all major indexing services

- Maximum visibility for your research

Submit your manuscript at www.biomedcentral.com/submit
(O) BioMed Central 